\title{
DE LA TRADICIÓN Y LA EVOLUCIÓN: BIENESTAR PSICOLÓGICO EN HOMBRES PATAGÓNICOS CONTEMPORÁNEOS
}

\author{
SEBASTIÁN PALLAHUALA U., SERGIO AVENDAÑO A. Y CLAUDIA ESTRADA G.
}

\begin{abstract}
RESUMEN
Lo masculino es un concepto fuertemente dependiente de las expectativas de roles, que trasciende las peculiaridades de una cultura local. En este contexto el presente estudio se ocupa de comprender las diferencias existentes entre hombres magallánicos de dos generaciones con respecto a sus concepciones de lo masculino, y la relación entre esas variables y el bienestar psicológico subjetivo. Un grupo de 80 personas del género masculino participaron contestando dos cuestionarios. Los resultados indican que las diferencias, tanto en el estereotipo de género, como en el bienestar psicológico no se superponen a estas agrupaciones etáreas, sino entre aquellos que adhieren y no a esquemas de género más y menos tradicionales. Aquellos hombres que muestran una integración en la identidad masculina entre los aspectos tradicionalmente masculinos y femeninos, muestran mejores niveles de bienestar que aquellos altamente masculinos, altamente femeninos o ninguno de los anteriores. Los resultados son discutidos en torno a la evolución del concepto de masculinidad, sus relaciones con vida moderna y la universalidad de este fenómeno.
\end{abstract}

PALABRAS CLAVE: identidad masculina, estereotipo de género, bienestar psicológico.

\section{FROM TRADITION AND EVOLUTION: PSYCHOLOGICAL BEING WELL IN CONTEMPORARY PATAGONIAN MEN}

\begin{abstract}
Masculinity is a concept that strongly depends on role expectations which transcends the peculiarities of local culture. This research studies the differences that exist between magellanic's men who belong two generations. Regarding their conceptions of masculinity and the relationship between these variables and subjective psychological well-being. A group of 80 male participants to answered two questionnaires. The results indicate that differences in both gender stereotype and psychological well-being, do not superimpose onto these age groups, but between those who adhere to it and not to more or less
\end{abstract}


traditional schemes. Those men who show an integration of male identity between male and female aspects, show higher levels of welfare than those strongly masculine, strongly feminine or neither to the former. The results are discussed according the evolution of the concept of masculinity, its relationship to modern life and the universality of this phenomenon.

KEY WORDS: male identity, gender stereotype, psychological well-being.

\section{INTRODUCCIÓN}

A lo largo de la historia han surgido diversos intentos teóricos por abordar la masculinidad y feminidad, suscitando especial interés la posición que lo masculino y lo femenino ocupa en el ordenamiento social y las relaciones de poder derivadas de dichas posiciones. Aunque los principales cambios en la concepción social de los géneros fueron impulsados por movimientos asociados al feminismo, este fenómeno implicó también la revisión de la masculinidad tradicional (Burin \& Meler, 2000). Algunas investigaciones, abandonando la mirada de la masculinidad como una construcción intocable, comenzaron a interesarse en el estudio del "malestar masculino" (Badinter, 1993; Burin, 2000; Montecino, 1998; Bonino, 1999).

Si bien, parece existir acuerdo respecto a que la diferenciación de roles es universal, la masculinidad y feminidad no se dan de la misma forma en todas las culturas, pues las ideas sobre lo que es considerado masculino y femenino en una cultura, corresponden a construcciones histórico-sociales, que se configuran en base a patrones relacionales entre los géneros. A pesar de las particularidades de cada sociedad y tiempo histórico, el valor predomínate en la cultura occidental, es que la masculinidad ha adoptado el rol dominante y la feminidad el de subordinación (Connell, 1997; Butler, 2001).

El concepto de género fue abordado inicialmente por autores tales como John Money (1955) quien postuló la noción de roles de género para referirse a las conductas que normalmente se le atribuyen a hombres y a mujeres, dando especial relevancia al contexto social y familiar en la configuración de la subjetividad genérica de las personas (Dio-Bleichmar, 1996; Klein, s.f; Botello, 2005).

Dado que por mucho tiempo se consideró que las nociones de género y sexo eran equivalentes, Stoller (1968) se dio al trabajo de delimitar tales conceptos. El sexo correspondería a la diferencia- ción que se realiza en función de las características sexuales anatómicas existentes entre hombres y mujeres, mientras que el género correspondería al significado atribuido a dicha diferencia, proveniente de construcciones sociales, culturales e históricas (Hardy y Jiménez, 2001; Carril, 2000; Burin, 1996).

Algunos autores plantean que el proceso de construcción de la subjetividad genérica comienza incluso antes de que el niño nazca (Hardy y Jiménez, 2001, Dio-Bleichmar, 1996, Quirici, 2003). Cuando los padres planifican la concepción de un hijo o durante el periodo de embarazo, éstos empiezan a fantasear respecto a las características que su hijo poseerá y sobre el sexo que dicho ser tendrá, identificando proyectivamente sobre el cuerpo sexuado de su hijo sus propios fantasmas de género.

Desde la mirada psicoanalítica, el periodo pre-edípico es el momento del desarrollo en el cual el sujeto comienza a identificarse con lo masculino. Estudios en torno a la identidad genérica han señalado que aproximadamente a los dieciocho meses de vida, el niño logra concebirse a sí mismo como perteneciente a un género, consolidándose lo que ha sido denominado como núcleo de género, antes de que éste sea capaz de comprender con total claridad la diferencia anatómico-genital existente entre los sexos (Stoller, 1968). En un primer momento la dependencia respecto de la madre es intensa, pues ella es la que se ha encargado tradicionalmente de cubrir las necesidades alimenticias y afectivas del bebé, sin embargo, cuando el padre se incorpora en la labor de crianza, éste emerge como principal modelo de identificación con lo masculino, siendo posicionado en el lugar de ideal (Dio-Bleichmar, 1996). Por lo tanto, el niño mantendrá un interés especial hacia el padre, que moviliza la identificación con él, no obstante esta actitud no se orienta hacia fines pasivos femeninos respecto del padre, pues a diferencia del vínculo que mantiene con la madre está exento del componente sexual; este proceso ha sido denominado como identificación primaria (Freud, 1921). 
Como se sabe, este vínculo simultáneo, de amor hacia la madre y de admiración hacia el padre, logra coexistir durante un tiempo, hasta que el niño, producto de los logros cognitivos del desarrollo, es capaz de comprender, de forma parcial, la diferencia anatómica entre los sexos y el significado del concepto marido-mujer, y los intercambios que se dan en la relación marital, lo cual da lugar al conflicto edípico. En este punto el niño percibe al padre como un obstáculo para acceder a la madre, lo cual genera su resentimiento, provocando que el vínculo con la figura paterna quede impregnado de componentes hostiles, producto de la rivalidad por la exclusividad materna, irrumpiendo la fantasía de destruirlo para poder ocupar su lugar. A pesar de la rivalidad existente entre hijo y padre, este último continua siendo idolatrado por el niño, el que lo percibe como un sujeto poderoso, que posee la potencia de la cual él carece y que le impide conservar a la madre (Bleichmar, 2006). Al respecto hay autores, que apoyándose en estudios antropológicos y en experiencias clínicas, han planteado que para que el niño pueda mantener la identificación con el padre durante el periodo edípico, es necesaria la existencia de un vínculo amoroso entre ambos, al margen de la rivalidad, donde la adopción de una actitud pasiva frente al padre, o un referente masculino equivalente, le transmitiría la potencia necesaria para consolidar su masculinidad, lo cual podría explicar la angustia homosexual dominante en el hombre (Bleichmar, 2006; Badinter, 1993).

Este periodo culmina cuando el padre establece la prohibición; impidiendo la fusión entre madre e hijo, lo cual conduce al niño a renunciar al amor hacia su madre como objeto de deseo incestuoso, bajo la amenaza de castración (Arvelo, 2001). En base a esta prohibición, se estructura la instancia superyoica, asociada a la conciencia moral, que se manifiesta por medio de la auto-observación y el control. En la disolución del complejo de Edipo, una de las metas es que el niño logre identificarse con el padre, quien posee las características que lo han convertido en el objeto de amor de la madre. La instancia superyoica controla la energía libidinal y orienta al sujeto al logro de una identidad que se asemeje al Ideal del yo, que en el caso específico de lo masculino, estaría conformado por las características del padre idealizado que han sido introyectadas por el niño.
Existen planteamientos teóricos que en el intento de delimitar el ideal masculino han establecido cinco pilares en los que se sustentaría la masculinidad tradicional, basándose en un ideal de autosuficiencia, que contempla un posicionamiento social y subjetivo de dominio y control (Bonino, 1999). El primer pilar, hace referencia a la hipótesis de que lo masculino se da por medio del distanciamiento de todo lo típicamente femenino (Inda, 1996; Badinter, 1993), negando la emocionalidad, la vulnerabilidad, las conductas de cuidado, la pasividad, entre otros. Esto surgiría como derivado de la dependencia materna original, repudiándose el deseo de fusión con ésta, frente al temor de la feminización, sentándose así las bases de actitudes misóginas y homofóbicas (Assoun, 2006). El segundo pilar está basado en la hipótesis de que la masculinidad se da por medio de la identificación con el padre idealizado, lo cual conllevaría al sujeto a buscar ser una persona importante. Este pilar se establece gracias al ejercicio del poder y la potencia, donde el éxito, la competitividad, la posición socioeconómica, el dominio y la búsqueda de la admiración de los otros, serian centrales en el ideal masculino tradicional. El tercer pilar, establece que la masculinidad se estructura y apoya en la rivalidad con el padre, que lleva al sujeto a acentuar los rasgos de dureza y distanciamiento afectivo (Burin, 2000; Badinter, 1993), que es lo que los llevaría a soportar todo tipo de aflicción, tolerando incluso padecimientos físicos. En torno a esto, la racionalidad se consolida como un rasgo importante, que le aseguraría al varón control e independencia respecto de los otros. El cuarto pilar establece que la masculinidad se construye sobre la base de la violencia, donde el tomar riesgos, resolver los conflictos utilizando la agresividad y la insensibilidad al sufrimiento de los otros fortalecería el ideal viril. Desde la mirada psicoanalítica, esto estaría motivado por la sublevación que generaría la amenaza de castración (Assoun, 2006), es decir, surge como una actitud reactiva debido a la sensación de pérdida de una posición de poder. El último pilar se sustenta en la idea de que los varones estarían orientados a respetar la jerarquía y los valores grupales masculinos, de esta forma los hombres tenderían a la homogeneización, como una forma de satisfacer su necesidad de pertenencia y de confirmación de una masculinidad débil (Inda, 1996). 
Por lo tanto, en nuestra cultura se le confiere al género masculino cualidades que alimentan un ideal heroico, altamente funcional y autovalente, lo cual le otorga un lugar de privilegio en el contexto social (Inda, 1996). Lo anterior hace suponer que ser hombre no constituye una posición desfavorable, sin embargo, las expectativas del rol genérico tradicional pueden significar muchas veces la percepción de demandas que requieren ser satisfechas por el individuo, que se acompañan de altos niveles de malestar, frente a la dificultad para responder a dichas exigencias. El ideal de autosuficiencia en la que se basa la masculinidad tradicional, suele armonizar con el auto concepto de forma positiva, pero puede oponerse a la salud mental o a la integridad física (Inda, 1996; Del Castillo, 2001; Olavarría, 2004; Hardy y Jiménez, 2001; Carril, 2000, Quirici, 2003).

Desde la psicología positiva han surgido diversos intentos de dar sentido y explicar un tema tan complejo como lo es la satisfacción que las personas experimentan en relación a sus vidas. Siendo conceptualizado de diversas formas, tales como felicidad, calidad de vida, bienestar subjetivo y bienestar psicológico (Barrientos, 2005).

Ryff (1989, en Casullo, 2002; Díaz et al, 2006) sugiere un modelo multidimensional de bienestar psicológico que se encuentra constituido por cinco dimensiones, dentro de las que se contemplan el control de situaciones, la aceptación de sí mismo, vínculos psicosociales, autonomía y proyectos. Además, algunos autores agregan al modelo la dimensión felicidad, como una dimensión que hace referencia a un estado psicológico, que contempla una aspecto emocional y otro cognitivo, que se configuraría como un factor protector frente al surgimiento de fenómenos psicopatológicos (Moyano y Ramos, 2007). El control de las situaciones, es una de las medidas de funcionamiento psicológico positivo que denota una capacidad para manejar de forma efectiva lo factores del medio ambiente y la propia vida, que se manifiesta en el sujeto por medio de la habilidad para producir cambios en su entorno y poder de esta forma asegurar la satisfacción de sus necesidades y motivaciones (Díaz et al., 2006; Castro, 2002). Las personas con un mayor control sobre las situaciones tienen una sensación de auto competencia elevada y son capaces de tolerar sus fracasos. La dimensión de aceptación de sí mismo, se caracteriza por una apreciación positiva de sí mismo y aceptación de todos los aspectos que componen al sujeto tanto positivos como negativos (Díaz et al., 2006). Una persona con una alta aceptación de sí mismo muestra conformidad con su cuerpo, con su forma de ser, con su pasado y acepta sus equivocaciones, sintiéndose impulsado a mejorar (Castro, 2002). La dimensión de vínculos psicosociales se relaciona con la capacidad para construir relaciones de confianza con otras personas y tener la capacidad de ser empáticos y afectivos. Además, el tener un buen numero de relaciones y una conexión con redes de apoyo a las cuales acudir frente a dificultades (Castro, 2002). Con relación a la dimensión de autonomía, esta se expresa por medio de la capacidad para tomar decisiones de forma independiente, resistiéndose en mayor medida a la influencia grupal, expresando lo que se piensa y autorregulando la propia conducta. Respecto a la dimensión de proyectos esta se manifiesta por medio del sentir de que la vida tiene propósito y significado, pues el tener metas y proyectos le otorga estructura y sentido la vida (Barrientos, 2006).

La evidencia señala que el conjunto de estas dimensiones no se dan de forma aislada sino que interactúan con contextos más amplios, como lo son los contextos microsocial y macrosocial, de esta forma emerge el concepto de dominios de satisfacción, dentro de las cuales se suelen identificar el área laboral, el área de las relaciones interpersonales, el área material y el área subjetiva (Sanchez-Cánovas, 1998).

Entonces, de forma general se puede definir el bienestar psicológico como el grado en el que una persona juzga en términos favorables su vida, asociado principalmente a la sensación de control percibido sobre las situaciones, la aceptación de sí mismo, la satisfacción con los logros alcanzados, la capacidad de establecer vínculos psicosociales satisfactorios y sentido de autodeterminación o autonomía, que pueden darse dentro de los dominios laboral, material, subjetivo y relaciones interpersonales.

Como un intento de conectar las formulaciones teóricas existentes respecto a la masculinidad tradicional con el concepto de bienestar psicológico, se podría hipotetizar que una persona satisfecha con su vida se caracterizaría por reconocer sus emociones y tener un amplio repertorio afectivo que es exteriorizado libremente (Barra, 2003). Debido a que la expresión de las emociones ha sido 
depositada tradicionalmente en las mujeres, aquellos hombres que adhieren al estereotipo masculino se ven impulsados a mantener un autocontrol constante de sus emociones, impidiendo la exteriorización de sentimientos dolorosos, de tristeza, de miedo, de ternura, amor y placer (Olavarría, 2004). El pobre manejo de las emociones podría generar dificultades al momento de identificarlas y/o expresarlas. Estas dificultades podrían traducirse en complicaciones para descifrar los estados internos de los otros, afectando su empatía y su capacidad para establecer relaciones significativas con otras personas.

La cuestión del poder y del dominio son aspectos sobre los que socialmente se ha construido lo masculino. El trabajo se ha conformado como una instancia en la cual el hombre logra validar su masculinidad, pues la actividad productiva lo posiciona como proveedor económico, necesario para mantener su dominio en el contexto familiar, situación que se ha visto desestabilizada con el ingreso de las mujeres al contexto laboral. Esta nueva realidad no solo entra en conflicto con los valores tradicionales sino que además, puede generar temor a la pérdida de una posición privilegiada y a fracasar en la búsqueda del éxito (Hardy y Jiménez, 2001).

Investigaciones señalan que una persona que tiene un grado elevado de satisfacción con su vida, aparte de tener una red social amplia y una buena integración social, es capaz de establecer mayor intimidad con las personas que le rodean. Los hombres que responden al estereotipo tradicional de masculinidad se muestran más resistentes a desarrollar una mayor intimidad en las relaciones con otras personas pues eso les significa mostrarse vulnerables, acercándose a una posición de dependencia respecto del otro, surgiendo el temor a la feminización (Hardy y Jiménez, 2001).

Debido a que los valores masculinos tradicionales sugieren que los hombres deben soportar las dificultades, la capacidad para detectar signos precoces de enfermedad se encuentra disminuida, además solicitar ayuda especializada supone aceptar la propia vulnerabilidad, lo cual es una muestra de debilidad a la cual los hombres suelen resistirse (Inda, 1996). Estudios han mostrado que los hombres tienen una percepción de salud mayor que las mujeres, lo cual se explica bajo la premisa de que las mujeres han sido socializadas para expresar su malestar, situación que no sucede con los hombres, quienes tienden a no exteriorizar y/o negar su malestar (Moyano y Ramos, 2007).

Las definiciones sobre la masculinidad se basan en ideales relacionadas con lo físico donde se privilegia la fuerza, la voluntad, el poder y la potencia sexual. Los hombres, teniendo en cuenta estas exigencias, se ven impulsados a mantener este ideal heroico, fortaleciendo los rasgos de dureza, con predominancia de la violencia y la poca sensibilidad al sufrimiento, lo cual los expone a situaciones que amenazan su integridad física (Inda, 1996). Cuando estos ideales tienen poco sustento en la realidad, surge malestar y tensión frente a la distancia que hay entre el ideal y las posibilidades individuales de alcanzar la demanda. No obstante, el varón lucha por alcanzarlo, pues su no cumplimento significaría defraudar al otro y a sí mismo, admitiendo su falta (Inda, 1996). Esta falta podría impactar en la subjetividad del varón en términos de pérdida, la cual al no ser elaborada puede teñir su vida con sintomatología depresiva, símil de un duelo patológico (Carril, 2000). Esta situación se torna problemática debido a la tendencia social a considerar como parámetro de normalidad la masculinidad, pues las características que componen la representación tradicional de dicho género son principalmente positivas o deseables socialmente.

Han surgido planteamientos que sugieren que las personas que no están tipificadas de acuerdo a conceptos genéricos tradicionales y que pueden flexibilizar sus esquemas masculinos y femeninos, dependiendo de la exigencias de las situaciones a las que se enfrentan, suelen adaptarse mejor a la vida, pues son personas más creativas, productivas y exitosas (Bem, 1974). En este contexto surge el concepto de androginia, para referirse a personas que tienen esquemas genéricos más flexibles, que en las situaciones son capaces de utilizar recursos personales sin importar si estos difieren de lo que socialmente se ha considerado como típico de lo masculino o lo femenino. Experimentos han puesto de relieve esta situación, enfatizando la actitud que las otras personas tienen hacia los sujetos que muestran indistintamente tanto características femeninas como masculina, se observó que las personas solían mostrar más aceptación hacia los sujetos no tipificados de acuerdo al género, siendo percibidas como personas más atractivas y exitosas, sin embargo, cuando un hombre o una mujer mostraban de forma 
predominante características típicas del otro género, fueron más cuestionadas y percibidas como mas desajustadas (Aronson, 1984). Se ha considerado que gradualmente más hombres y mujeres desarrollan modelos genéricos más integrados y adaptativos, sin embargo, esto entra en conflictos con una sociedad en la que aun se privilegian los valores tradicionales, y que trasmite a los varones la importancia de ser un hombre importante, que tome riesgos, pero que se encuentre desconectado de sus afectos, que no muestre vulnerabilidad y necesidad de dependencia (Badinter, 1993; Burin, 2000).

Sobre la relación entre la adhesión a un esquema tradicional de masculinidad y el bienestar psicológico subjetivo es de lo que se ocupa el presente estudio. Busca realizar un acercamiento al estudio de género que compara los diferentes esquemas estereotípicos a los que adhieren los hombres patagónicos en sus niveles de satisfacción con su vida, en la intensión de aportar en la exploración de la situación actual de la masculinidad desde una cultura que, más allá de sus particularidades, comparte los roles de género definidos en el mundo occidental. El hombre patagónico chileno-magallánico, se ha construido, como sujeto, paralelamente a la historia de la ocupación de un territorio geográfico "difícil". Como nativo, inmigrante europeo o chilote, ha compartido la exigencia de verse enfrentado a duras condiciones climáticas y de aislamiento. En tal sentido, las tareas que ha desarrollado tradicionalmente, tales como labores en el campo (crianza de ganado ovino), pescador, en la industria petrolera, factorías industriales y mineras, han requerido de una exacerbación de los valores estereotípicos de la masculinidad tradicional (Low, 2001). Desde esta perspectiva, los hombres patagones son un buen ejemplo de "masculinidad tradicional", cuyos cambios y sus procesos son extrapolables a la cultura general.

\section{MÉTODO}

\section{Participantes y Diseño}

La muestra no probabilística accidental estuvo compuesta por 80 hombres magallánicos, de dos grupos edad. El primero compuesto por 40 hombres menores de 35 años y el segundo grupo por 40 hombres mayores de 35 años. La moda de escolaridad fue estudios universitarios incompletos.
Respecto al estado civil se observó que el 50\% de los participantes reportó estar soltero, el 40\% casado, el 10\% restante se declaró conviviendo, separado o divorciado. Se utilizó un diseño no experimental transversal correlacional.

\section{Instrumentos}

Escala de adhesión al estereotipo masculino tradicional: Escala destinada a medir la adscripción al estereotipo masculino tradicional, compuesta por dos partes. La primera consta de 40 rasgos pre-testeados, entre los cuales se debían elegir los 10 más propios de la masculinidad. La segunda consta de 20 afirmaciones sobre características que pueden o no asignarse a un hombre típico (sobre escala Likert de 5 puntos; 1: "Para nada" hasta 5: "Totalmente"). La mitad de estos ítems evalúan características pre-testeadas típicas masculinas mientras que los 10 restantes evalúan características femeninas. Los resultados de esta parte permitía dividir la muestra en cuatro grupos según su adhesión alta/baja al estereotipo femenino y alta/baja al estereotipo masculino. La consistencia interna de ambas dimensiones (femenina y masculina) fue superior a 0.65 (alfa).

Cuestionario de bienestar Psicológico: Cuestionario que mide el nivel de bienestar psicológico que las personas experimentan respecto de sus vidas. Fue construido integrando dos modelos distintos de bienestar; dominios de bienestar: subjetivo, laboral, relaciones interpersonales y material; y dimensiones de bienestar: control de situaciones, aceptación de sí mismo, vínculos psicosociales, proyectos, autonomía y felicidad. El instrumento compuesto por 44 ítems, en el que cada afirmación mide un dominio y una dimensión simultáneamente que se responden en base a una escala Likert de 5 puntos que va desde el 1:"Totalmente en desacuerdo" hasta el 5:"Totalmente de acuerdo".

\section{Procedimiento}

La aplicación se realizó de forma individual. Cada participante fue contactado en su lugar de estudio, trabajo o habitación. Aceptaron participar de forma voluntaria, firmaron un consentimiento informado y se auto-aplicaron los instrumentos. Al finalizar, se revisaron las posibles preguntas o reactivos 
sin contestar y se les agradeció por su participación. Cada participante recibió sus resultados individuales al momento de finalizar la recolección de datos.

\section{RESULTADOS}

La evaluación de los rasgos definidos como típicamente masculinos muestra que el estereotipo del hombre típico está constituido por los rasgos: valiente $(63,8 \%)$, trabajador $(62,5 \%)$, inteligente $(52,5 \%)$, fuerte $(52,5 \%)$ y protector $(51,3 \%)$. Los rasgos contra estereotípicos serían: célibe (0\%), desagradable $(1,3 \%)$, irresponsable $(3,8 \%)$, débil $(3,8 \%)$, cobarde (5,0\%), emotivo (6,3\%), dependiente $(8,8 \%)$, que promueve la igualdad $(8,8 \%)$ y pasivo (10\%). Los estereotipos no muestran diferencias significativas al ser comparados según grupo de edad.

Los resultados de la escala de adscripción al estereotipo masculino muestran que en la dimensión "masculinidad", el promedio del grupo fue de 31,11 , mientras que en la dimensión de "feminidad" el promedio del grupo fue de 27,48 (ambas con un máximo hipotético de 50). La frecuencia de los cuatro grupos de esquema de género (alto en ambas, bajo en ambas, bajo en masculinidad y alto en femineidad, $y$ alto en masculinidad y bajo en femineidad) indica que los grupos con mayor frecuencia fueron los que combinan alto en uno y bajo en otro. (Ver tabla 1)

Al comparar el puntaje promedio de la muestra $(M=4,15 ; D . E .=0,53)$ en Bienestar psicológico con el punto neutro de la escala, se observa una diferencia significativa que indica adecuados niveles de satisfacción, que se sitúan en el percentil $40\left(t_{(80)}=\right.$ 19,37, $p<0,0001)$. Al realizar el cálculo tanto para los dominios como para las dimensiones, los promedios de bienestar se sitúan por sobre el punto neutro de la escala. En relación al bienestar psicológico total según grupo de edad, no se observan diferencias significativas $\left(F_{(3,72)}=0,71\right.$, n.s. $)$, las que si surgen al comparar los 4 grupos de esquemas de género $\left(F_{(3,72)}=3,90, p<0,01\right)$. La principal diferencia se observa entre el grupo con un esquema predominantemente masculino $(M=3,93 ; D E=0,50)$, y el grupo alto en masculinidad y feminidad $(M=4,46$; $D E=0,49$ ), ya que los primeros muestran niveles de bienestar significativamente menores a los segundos. En términos globales, solamente el grupo con un esquema predominantemente masculino presenta un promedio que se sitúa fuera del rango
Tabla 1. Frecuencias de grupos según esquemas de género.

\begin{tabular}{ccc}
\hline & $\begin{array}{c}\text { Bajo en } \\
\text { Feminidad }\end{array}$ & $\begin{array}{c}\text { Alto en } \\
\text { Feminidad }\end{array}$ \\
\hline Bajo en Masculinidad & 16 & 24 \\
Alto en Masculinidad & 25 & 15 \\
\hline
\end{tabular}

de normalidad, es decir, bajo lo esperado (Pc: 20) (Ver figura 1).

$\mathrm{Al}$ analizar las dimensiones de bienestar psicológico según los grupos de esquemas de género, se observa que en la dimensión "felicidad", existen diferencias significativas $\left(F_{(3,72)}=6,12, p<0,001\right)$, particularmente entre los grupos con un esquema predominantemente masculino $(M=3,67 ; D E=0,68)$ $y$ aquellos con un esquema alto en masculinidad y feminidad $(M=4,47 ; D E=0,46)$, donde el último muestra tener un nivel de bienestar mayor que el primer grupo. También se observaron diferencias en la dimensión "proyectos" $\left(F_{(3.72)}=2,99, p<0,037\right)$. El grupo con un esquema alto en masculinidad y feminidad, muestra tener un nivel mayor de bienestar $(M=4,50 ; D E=0,48)$ en comparación al grupo de bajo en masculinidad y feminidad $(M=4,13 ; D E=$ $0,67)$. Finalmente en la dimensión "aceptación de sí mismo" el grupo con un esquema alto masculinidad y feminidad, muestra tener un mayor nivel de bienestar $(M=4,48 ; D E=0,60)$ en comparación al grupo que tiene un esquema predominantemente masculino $(M=3,80 ; D E=0,68),\left(F_{(3,72)}=5,70, p\right.$ $<0,001)$. Ni para la dimensión "autonomía", ni para

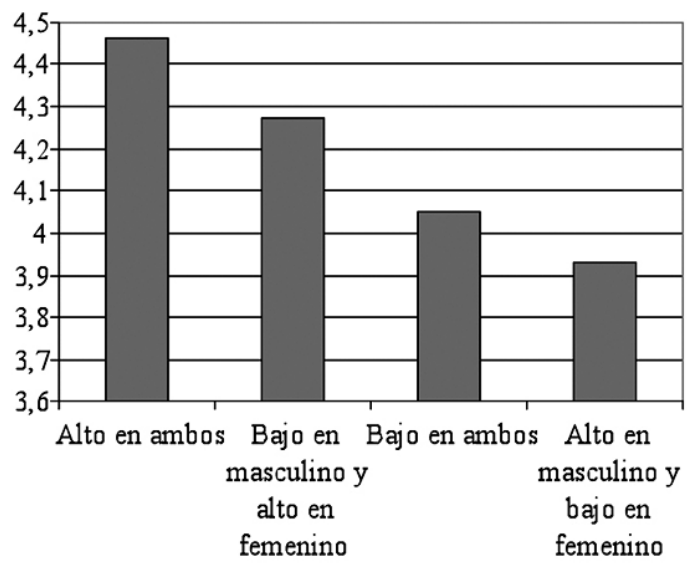

Fig. 1. Promedio de grupos de esquema genérico en relación al bienestar psicológico total. 
la de "vínculos psicosociales", ni para la de "control de situaciones" se aprecian diferencias entre los grupos de esquema de género.

$\mathrm{Al}$ analizar los resultados por dominios de bienestar también se encontraron diferencias significativas en relación a los grupos de esquemas de géneros. En el dominio "laboral" aquellos que presentan un esquema bajo en masculinidad y feminidad $(M=3,98 ; D E=0,60)$ y el grupo con un esquema predominantemente masculino $(M=3,82$; $D E=0,55)$ muestran menores niveles de bienestar que los participantes pertenecientes a los grupos con un esquema predominantemente femenino $(M=$ $4,16 ; D E=0,66)$ y el grupo con un esquema alto masculinidad y feminidad $(M=4,38 ; D E=0,55)$, $\left(F_{(3,72)}=2,96, p<0,038\right)$. En el dominio "subjetivo" el grupo que presenta un esquema predominantemente masculino $(M=3,91 ; D E=0,59)$, muestra menor bienestar que aquellos con un esquema alto masculinidad y feminidad $(M=4,52 ; D E=0,48),(F$ $(3,72)=4,04 ; p<0,010)$. Por último para el dominio de "relaciones interpersonales", se observa que los participantes del grupo con un esquema alto en masculinidad y feminidad muestran mayores niveles de bienestar $(M=4,57 ; D E=0,58)$ que el observado en el grupo con un esquema predominantemente masculino $(M=4,0 ; D E=0,57),\left(F_{(3,72)}=4,19, p\right.$ $<0,009)$. Para el dominio "material" no se aprecian diferencias entre los grupos de esquema de género.

\section{DISCUSIÓN Y CONCLUSIONES}

El propósito de éste estudio se centró en conocer la relación existente entre las variables adscripción al estereotipo tradicional de masculinidad y el bienestar psicológico en una muestra de hombres "magallánicos". Los resultados permitieron conocer el concepto actual que estos sujetos tienen sobre la masculinidad, constituido por rasgos deseables y rechazados. La hipótesis de investigación postuló que aquellos hombres que adhieren al estereotipo masculino, experimentan menores niveles de bienestar psicológico y viceversa. Los resultados confirman esta hipótesis, añadiendo un dato relevante que no fue contemplado inicialmente, que estriba en aquellos hombres que integran tanto aspectos masculinos como femeninos en su identidad genérica, reportan mayores niveles de bienestar que aquellos que tienen una identidad genérica predominantemente masculina.
Al solicitarles a los participantes que caracterizaran al hombre típico por medio de una selección de rasgos, fue posible construir un perfil del estereotipo, el cual estuvo compuesto por aquellas características que fueron altamente seleccionadas y las que fueron rechazadas. Se constató que los rasgos que son considerados como centrales del concepto, corresponden en su totalidad a aspectos que en las sociedades occidentales contemporáneas son valoradas de forma positiva o son socialmente deseables y que alimentan el ideal de autosuficiencia masculino (Bonino, 1999). Estereotipo que exige que los hombres sean capaces de tomar riesgos, anulando todo tipo de temores que revelen su vulnerabilidad, esto implica acentuar sus rasgos de dureza emocional, como requisito para poder consolidarse como una figura capaz de proteger y otorgar seguridad. A lo que se suma el orientarse al ejercicio de una función productiva que tenga como fin la validación social y que asegure el sustento familiar.

Entre los rasgos rechazados se ponen de relieve aquellos que cuestionan los pilares en los que se sustenta la masculinidad tradicional, adquiriendo especial relevancia lo relativo a la sexualidad, donde una actitud célibe emerge como una amenaza, pues la presencia de una vida sexual activa, algo disociada del componente emocional, sería un elemento importante para fortalecer el ideal viril basado en la potencia y el desempeño. Otros de los rasgos excluidos por los participantes fueron lo que se asocian a la dependencia, a la pasividad, la debilidad y la irresponsabilidad, que harían peligrar el ideal de autosuficiencia, del cual deriva una necesidad de autonomía, de productividad y eficiencia (Badinter, 1993). Por lo tanto, en los participantes se revela una alta adscripción al estereotipo de género presente en la cultura dominante occidental, no evidenciándose características especiales que los diferencien de otros hombres estudiados. Sin embargo, ello no necesariamente significa que el estereotipo local no pudiese diferir en cuanto a la intensidad o fuerza del mismo, cuestión que no fue evaluada en el presente estudio y que resta como una tarea para estudios futuros.

Si bien, los niveles de bienestar psicológico reportado por los participantes en cada una de las dimensiones y dominios mostraron estar, en su mayoría, dentro de rangos normales según las normas locales, resulta interesante constatar las diferencias entre los grupos con distintos esquemas genéricos. 
Dentro de los resultados más relevantes del estudio, se encuentra la tendencia de los hombres a experimentar mayor bienestar psicológico en las distintas áreas de su vida, al integrar como parte de su identidad genérica, rasgos que tradicionalmente se han considerado como típicamente femeninos. Aparentemente, cuando los hombres logran articular en su personalidad aspectos masculinos y femeninos, sin que estos entren en conflicto, son mejores al afrontar eficazmente las situaciones a las que se enfrentan en las diversas áreas de su vida. En la dirección de las investigaciones desarrolladas por autores tales como Bem (1974), este resultado podría revelar la presencia de un conjunto de estrategias más adaptativas para resolver los problemas, ya que ello implica mayor flexibilidad en la utilización de recursos cognitivos y afectivos, sin vivenciar esta situación como una amenaza a su masculinidad.

Las diferencias entre los grupos con diferentes esquemas genéricos en cuanto a la dimensión "felicidad", apunta a que la integración de aspectos tradicionalmente considerados femeninos, favorecen la conexión del sujeto con su mundo interior, específicamente con los afectos. Aquellos participantes con baja integración de lo femenino son menos felices, lo que podría explicarse por los requerimientos del esquema masculino en el que se teme la feminización y la pérdida de control racional, que atentaría con los cimientos de una subjetividad genérica rígida. Este resultado se ve reforzado por el observado en el dominio "subjetivo".

En relación a la dimensión de "proyectos", los resultados indican que aquellos individuos que adscriben al estereotipo tradicional masculino reportan menores niveles de bienestar, siendo los únicos que se sitúan bajo lo esperado, lo que contrasta con quienes no presentan tal rasgo. De acuerdo al modelo masculino tradicional, el tener metas, aspiraciones $y$ proyectos en la vida, es un aspecto importante, pues el imperativo social sugiere que el hombre debe o debería estar orientado al logro, lo que da lugar no solo a la generación de metas sino también la implementación de las estrategias necesarias para alcanzarlas. No es infrecuente que los sujetos se muestren mas orientados a validarse sobre la base de expectativas de orden social, que a satisfacer sus genuinas necesidades y aspiraciones personales. Cuestión que presupone una adecuada integración de la emocionalidad, lo que implica armonizar as- pectos considerados como típicamente masculinos con otros considerados como típicamente femeninos. El alcanzar estas metas "sociales", postergando las necesidades personales, también puede asociarse a la problemática masculina de la búsqueda del éxito, donde la ejecución de las acciones necesarias para conseguir tales objetivos puede generar una gran sobrecarga psíquica, derivado del miedo al fracaso, que puede desembocar incluso en manifestaciones físicas, como suele suceder con los problemas cardiacos (Bonino, 1999).

De acuerdo a la dimensión "aceptación de sí mismo", se considera que es necesario aceptar no sólo los aspectos positivos que forman parte de la vida del individuo sino que también se requiere el reconocimiento e integración de los aspectos negativos y la aceptación de los propios errores. Los resultados sugieren que los hombres que adscriben al estereotipo masculino tradicional, posiblemente tiendan a pasar por alto los aspectos negativos de su vida, pues esto pondría en evidencia sus puntos débiles, revelando una especie de artefacto defectuoso, lo que constituiría un riesgo para la integridad del ideal omnipotente de la masculinidad tradicional. En consecuencia, el resultado que indica un menor bienestar de este grupo en dicha dimensión, también confirma nuestra hipótesis de trabajo.

En el contexto laboral, también se reproducen los resultados anteriores, es el área donde el ideal viril tradicional, sugiere la imagen de un hombre que sea capaz de adoptar eficientemente el rol de proveedor, lo que conlleva a que los individuos inviertan altos niveles de energía en su desempeño en este dominio, con el propósito de asegurar el sustento familiar. Si bien el trabajo alimenta una imagen productiva valorada socialmente, la energía desplegada en la ejecución de estas actividades produce un desgaste psíquico considerable, que pone en entredicho el bienestar del sujeto, al postergar momentos y actividades orientadas al autocuidado.

En el ámbito de las relaciones "interpersonales", el hecho de que las personas que parecen tener un esquema rígidamente masculino muestren menores niveles de bienestar que aquellos que poseen un esquema más flexible o integrador respecto al par masculino/femenino, podría explicarse en base al compromiso emocional que las relaciones cercanas implican, donde el establecimiento de un vínculo recíproco entre sujetos, que incluye la aceptación 
de la propia vulnerabilidad y la necesidad de otros. Estos rasgos son rechazados, pues constituye un aspecto contradictorio respecto del ideal masculino tradicional, dado que este ideal se caracteriza por obligar a invertir un esfuerzo considerable en acentuar los rasgos de dureza y de distancia respecto de los demás, buscando el establecimiento de una relación desigual de dominación-sumisión, donde la intimidad queda postergada, mecanismo mediante el cual la amenaza de feminización y pasividad, derivada de la actualización de la dependencia materna, es negada (Bleichmar, 2006). Si bien esta actitud de distancia respecto de los otros, puede ser útil para mantener intacto el ideal masculino, ello puede generar una disminución del bienestar psicológico, al encontrarse el sujeto carente de vínculos significativos, por lo tanto, aparentemente la presencia de un esquema de género más flexible, que implica dinámicas interpersonales de mayor cercanía afectiva tradicionalmente atribuido a lo femenino, puede orientar al sujeto a establecer lazos significativos con las otras personas en las distintas dimensiones de su vida. Elemento relacional que conformaría un factor protector frente a situaciones potencialmente patógenas.

Los resultados obtenidos permiten hacer algunas inferencias interesantes sobre el concepto que el hombre patagónico tiene sobre la masculinidad y sobre lo que se ha dado en llamar el "malestar masculino". Se observó que su noción de masculinidad no se diferencia de forma significativa con el estereotipo masculino tradicional ni existen diferencias importantes entre los dos grupos de edad estudiados, sin embargo, parece existir una tendencia a adherir a modelos genéricos menos tradicionales. La integración de rasgos típicamente femeninos se asoció a un aumento del bienestar psicológico, pero ¿Cuál es el impacto que esto tiene en el entorno social? ¿Cuál será el efecto que genera en las otras personas un hombre que muestra tener rasgos femeninos? en futuras investigaciones resultaría interesante poder analizar la actitud que las otras personas adoptan al relacionarse con hombres que integran aspectos femeninos en su identidad genérica, explorando la posible presencia de prejuicios explícitos o implícitos, lo que revelaría la forma en que el contexto social actual favorece o entorpece la generación de esquemas genéricos más flexibles o adaptativos.

\section{BIBLIOGRAFÍA}

ARONSON, E. 1984. El animal social: introducción a la psicología social. Editorial Alianza, Madrid.

ARVELO, L. 2001. Masculinidad y función paterna. Otras miradas $1(1): 43-52$.

ASSOUN, P. 2006. Lecciones psicoanalíticas sobre masculino y femenino. Editorial Nueva Visión, Buenos Aires.

BADINTER, E. 1993. XY, La identidad masculina. Editorial Alianza, Madrid.

BARRA, E. 2003. Influencia del estado emocional en la salud física. Terapia Psicológica 21:55-60.

BARRIENTOS, J. 2005. Calidad de vida, bienestar subjetivo:Una mirada psicosocial. Universidad Diego Portales, Santiago.

BEM, S.L. 1974. The measurement of psychological androgyny. Journal of consulting and clinical Psychology. American Psychological Association 42(2):155-162.

BLEICHMAR, S. 2006. Paradojas de la sexualidad masculina. Editorial Paidós, Buenos Aires.

BONINO, L. 1999. Varones, género y salud mental: desconstruyendo la normalidad masculina. En: Nuevas masculinidades. Editores Segarra, M. y Carabí, A. (eds) 2000 Icaria. Barcelona.

BOTELLO, L. 2005. Identidad, masculinidad y violencia de género. Memoria para optar al grado de Doctor. Universidad complutense de Madrid. Madrid.

BURIN, M. 2000. Construcción de la subjetividad masculina. En: Varones: Género y subjetividad masculina. Editores Burin, M y I. Miller. Editorial Paidós, Buenos Aires.

BURIN, M. y I. MELER 2000. Atendiendo el malestar de los varones. En: Varones: Género y subjetividad masculina. Editores Burin, M y I. Miller. Editorial Paidós, Buenos Aires.

BURIN, M. y I. MELER 2000. Género: una herramienta teórica para el estudio de la subjetividad masculina. En: Varones: Género y subjetividad masculina. Editores Burin, M y I. Miller. Editorial Paidós, Buenos Aires.

BUTLER, J. 2001. El género en disputa. Editorial Paidós, México.

CARRIL, E. 2000. Femenino/masculino. La pérdida de ideales y duelo. En: Los duelos y sus Destinos. Depresiones, hoy. Montevideo: Asociación Psicoanalítica del Uruguay, Uruguay.

CASULLO, M. 2002. Evaluación del bienestar psicológico en Iberoamérica. Editorial Paidós, Buenos Aires.

CASTRO SOLANO, A. 2002. Investigaciones argentinas sobre el bienestar psicológico, En: Evaluación del bienestar psicológico en Iberoamérica. Editores Casullo, M (Comp.) Editorial Paidós, Buenos Aires. 
CONNELL, R 1997. La organización social de la masculinidad. En: Masculinidad/es: Poder y crisis. Editores Valdés, T. y J. Olavarría. Ediciones de la mujer, 24. Isis internacional/ FLACSO, Santiago.

DEL CASTILLO, D. 2001. Los fantasmas de la masculinidad. En: http://www.mineduc.cl/usuarios/edu.sexual/ doc/200608211808040.Identidad\%20Masculina\%20 y\%20Femenina.pdf.

DÍAZ, D., R. RODRÍGUEZ-CARVAJAL, A. BLANCO, B. MORENO-JIMÉNEZ, I. GALLARDO, C. VALLE y D. VAN DIERENDONCK 2006. Adaptación española de las escalas de bienestar psicológico de Ryff. Psicothema 18(3):572-577. Austria.

DIO-BLEICHMAR, E. 1996. Feminidad-masculinidad. Resistencias en el psicoanálisis al concepto de género. En: Género, psicoanálisis, subjetividad. Editores Burin, M y Dio-Bleichmar, E. (Comp.). Editorial Paidós, Buenos Aires.

ESTRADA, C., C. SALVATORRE, E. TORRES y S. VALDERRAMA 2005. Manual Operativo de Metodología Cuantitativa. FONAD 2005, Universidad de Magallanes, Punta Arenas.

FREUD, S. 1921. Psicología de las masas y análisis del yo. En: Obras Completas. Amorrortu, Barcelona.

HARDY, L. Y JIMÉNEZ A. 2001. Masculinidad y género. Revista cubana de salud pública. 27(2):77-88.

INDA, N. 1996. Género masculino, número singular, En: Género, psicoanálisis, subjetividad. Editores Burin, M y E. Dio-Bleichmar (Comp.), Editorial Paidós, Buenos Aires.

KLEIN, R (s.f.) El nacimiento del género. Colofón, 22.

LOW, K. 2001. Construcción de la masculinidad entre ovejeros de la estancia magallánica. Tesis no publicada para la obtención del título de licenciado en antropología. Universidad de Chile, Santiago.

MOYANO, E. y RAMOS, N. 2007. Bienestar subjetivo: midiendo satisfacción vital, felicidad y salud en población chilena de la región del Maule. Universum, 22(2):177-193. Talca.

MONTECINO, S. 1998. De lachos a machos tristes: la ambivalencia de lo masculino en Chile. En: Dialogo sobre el género masculino en Chile. Editores Montecino, S. y M. E. Acuña, Colección de libros electrónicos - Serie estudios, Santiago.

MONEY, J., J.G. HAMPSON y J.L. HAMPSON 1955. Hermaphroditism: Recommendations concerning assignment of sex, change of sex, and psychological management. Bulletin of the John Hopkins Hospital 97: 284-300.

OLAVARRÍA, J. 2004. Invisibilidad y poder. Varones en Santiago de Chile. En: Hombres e identidades de género. Investigaciones desde América Latina. Editores Viveros, M, J. Olavaria y M. Fuller (Comp.). Universidad nacional de Colombia, Bogotá.

SÁNCHEZ-CÁNOVAS, J. 1998. EBP. Escala de bienestar psicológico. TEA Ediciones, Madrid.

STOLLER, R. 1968. Sex and Gender. Jason Aronson. Nueva York.

QUIRICI, T. 2003. ¿El género hace el síntoma? Masculinidad y trastornos obsesivos. Revista de psicoterapia psicoanalítica. 3:57-68.

TAJFEL, H. 1984. Grupos humanos y categorías sociales. Herder. Barcelona.

VIVEROS, M. 2001. Masculinidades, diversidades regionales y cambios generacionales En: Hombres e identidades de género. Investigaciones desde América Latina. Universidad Nacional de Colombia. Colombia. 
